\title{
AXISYMMETRIC LAMB'S PROBLEM IN A SEMI-INFINITE MICROPOLAR VISCOELASTIC MEDIUM
}

\author{
P.K. BISWAS \\ P O Parbatipur \\ Dist - NADIA \\ West Bengal, INDIA PIN 741235
}

\author{
P.R. SENGUPTA \\ Department of Mathematics \\ Kalyani University \\ Kalyani, West Bengal, INDIA
}

\author{
and \\ LOKENATH DEBNATH \\ Department of Mathematics \\ University of Central Florida \\ Orlando, Florida 32816, U S A
}

(Received July 6, 1994 and in revised form September 10, 1995)

\begin{abstract}
A study is made of the axisymmetric problem of wave propagation under the influence of gravity in a micropolar viscoelastic semi-infinite medium when a time varying axisymmetric loading is applied on the surface of the medium Special attention is given to the effects of gravity which induces a kind of initial stress of a hydrostatic nature on the wave propagation
\end{abstract}

KEY WORDS AND PHRASES: Axisymmetric Lamb's Problem, micropolar viscoelastic medium, and wave propagation

1991 AMS SUBJECT CLASSIFICATION CODES: 73D

\section{INTRODUCTION}

In classical problems of wave propagation in an elastic medium studied by several authors including Love [1] and De and Sengupta [2], it has been shown that the velocity of Rayleigh waves increases by a significant amount when the wave-length is large due to the influence of gravity Biot [3] investigated the influence of gravity on Rayleigh waves under the assumption that the force of gravity generates an initial stress of a hydrostatic nature so that the medium remains incompressible Nowacki and Nowacki [4] discussed the axisymmetric Lamb's problem in a semi-infinite micropolar elastic solid However, they did not include the effects of gravity in a micropolar viscoelastic solid medium The main purpose of this paper is to consider the axisymmetric Lamb's problem in a semi-infinite micropolar viscoelastic medium under the influence of gravity due to a harmonically oscillating loading acting on the surface of the medium Special attention is given to the effects of gravity which generates an initial stress hydrostatic in nature, on the wave propagation

\section{FORMULATION OF THE PROBLEM}

We consider a viscoelastic homogeneous isotropic centrosymmetric body and assume that the initial stress due to gravity is hydrostatic in nature Since the initial stress is hydrostatic, stress strain relations 
in this case will remain the same as in a medium initially stress free The stress and strain relations in the micropolar viscoelastic medium are

$$
\begin{aligned}
& \sigma_{\jmath \iota}=\left\{\left(\mu_{0}+\alpha_{0}\right)+\left(\mu_{1}+\alpha_{1}\right) \frac{\partial}{\partial t}\right\} \nu_{\jmath \imath}+\left\{\left(\mu_{0}-\alpha_{0}\right)+\left(\mu_{1}-\alpha_{1}\right) \frac{\partial}{\partial t}\right\} \nu_{\imath \jmath}+\left(\lambda_{0}+\lambda_{1} \frac{\partial}{\partial t}\right) \nu_{k k} \delta_{\iota \jmath} \\
& \mu_{\jmath \prime}=\left\{\left(\nu_{0}+\epsilon_{0}\right)+\left(\nu_{1}+e_{1}\right) \frac{\partial}{\partial t}\right\} \chi_{\jmath \imath}+\left\{\left(\nu_{0}-\epsilon_{0}\right)+\left(\nu_{1}-\epsilon_{1}\right) \frac{\partial}{\partial t}\right\} \chi_{\iota \jmath}+\left(\beta_{0}+\beta_{1} \frac{\partial}{\partial t}\right) \chi_{k, k} \delta_{\iota \jmath} \\
& \nu_{\jmath \prime}=u_{\imath, \jmath}-e_{k \jmath} \omega_{k}, \quad \chi_{\jmath 2}=\omega_{\iota, \jmath}
\end{aligned}
$$

where $\lambda_{0}, \mu_{0}, \alpha_{0}, \beta_{0}, \nu_{0}, \epsilon_{0}$ are elastic parameters, $\lambda_{1}, \mu_{1}, \alpha_{1}, \beta_{1}, \nu_{1}, \epsilon_{1}$ are the parameters associated with viscosity

We use the cylindrical polar coordinates $(r, \theta, z)$ Without body couples, external loading distributions, body forces, the displacement vector $\mathbf{u}$, rotation vector $\omega$ depend only on $r, z$ and $t$ because of the axisymmetric configuration The equations of motion in a micropolar viscoelastic solid medium under the influence of gravity are given by

$$
\begin{aligned}
& {\left[\left(\mu_{0}+\alpha_{0}\right)+\left(\mu_{1}+\alpha_{1}\right) \frac{\partial}{\partial t}\right]\left(\nabla^{2} u_{r}-\frac{u_{r}}{r^{2}}\right)+\left[\left(\lambda_{0}+\mu_{0}-\alpha_{0}\right)+\left(\lambda_{1}+\mu_{1}-\alpha_{1}\right) \frac{\partial}{\partial t}\right] \frac{\partial e}{\partial r}} \\
& -2\left(\alpha_{0}+\alpha_{1} \frac{\partial}{\partial t}\right) \frac{\partial \omega_{\theta}}{\partial z}+\rho g \frac{\partial u_{z}}{\partial r}=\rho u_{r} \\
& {\left[\left(\mu_{0}+\alpha_{0}\right)+\left(\mu_{1}+\alpha_{1}\right) \frac{\partial}{\partial t}\right] \nabla^{2} u_{z}+\left[\left(\lambda_{0}+\mu_{0}-\alpha_{0}\right)+\left(\lambda_{1}+\mu_{1}-\alpha_{1}\right) \frac{\partial}{\partial t}\right] \frac{\partial e}{\partial z}} \\
& +\frac{2\left(\alpha_{0}+\alpha_{1} \frac{\partial}{\partial t}\right)}{r} \frac{\partial}{\partial r}\left(r \omega_{\theta}\right)-\rho g \frac{1}{r} \frac{\partial}{\partial r}\left(r u_{r}\right)=\rho u_{z} \\
& {\left[\left(\nu_{0}+\epsilon_{0}\right)+\left(\nu_{1}+\epsilon_{1}\right) \frac{\partial}{\partial t}\right]\left(\nabla^{2} \omega_{\theta}-\frac{\omega_{\theta}}{r^{2}}\right)-4\left(\alpha_{0}+\alpha_{1} \frac{\partial}{\partial t}\right) \omega_{\theta}} \\
& +2\left(\alpha_{0}+\alpha_{1} \frac{\partial}{\partial t}\right)\left(\frac{\partial u_{r}}{\partial z}-\frac{\partial u_{z}}{\partial r}\right)=J \omega_{\theta}
\end{aligned}
$$

where

$$
e=\frac{1}{r} \frac{\partial}{\partial r}\left(r u_{r}\right)+\frac{\partial u_{z}}{\partial z}, \quad \nabla^{2}=\frac{\partial^{2}}{\partial r^{2}}+\frac{1}{r} \frac{\partial}{\partial r}+\frac{\partial^{2}}{\partial z^{2}}
$$

On the free surface $z=0$, the axially symmetrical and time varying loadings normal and tangential to the boundary surface and moment with a vector tangent to a circle of radius $r$ are applied The displacement components $u_{r}, u_{z}$ and rotation component $\omega_{\theta}$ are independent of $\theta$

We introduce a scalar potential $\phi$ and a vector potential $\psi$ and express the displacement components $u_{r}, u_{z}$ in terms of these potentials

$$
u_{r}=\frac{\partial \phi}{\partial r}+\frac{\partial^{2} \psi}{\partial z \partial r}, \quad u_{z}=\frac{\partial \phi}{\partial z}-\left(\frac{\partial^{2}}{\partial r^{2}}+\frac{1}{r} \frac{\partial}{\partial r}\right) \psi
$$

Introducing $\omega_{\theta}=-\frac{\partial \chi}{\partial r}$ and putting (2 7ab) into (2.4)-(26), we obtain the following set of wave equations

$$
\left[\nabla^{2}-\frac{1}{\left(c_{1}^{2}+c_{1}^{\prime 2} \frac{\partial}{\partial t}\right)} \cdot \frac{\partial^{2}}{\partial t^{2}}\right] \phi-\frac{g}{c_{1}^{2}+c_{1}^{\prime 2} \frac{\partial}{\partial t}}\left[\frac{\partial^{2}}{\partial r^{2}}+\frac{1}{r} \frac{\partial}{\partial r}\right] \psi=0
$$




$$
\left(\nabla^{2}-\frac{1}{c_{2}^{2}+c_{2}^{\prime 2} \frac{\partial}{\partial t}} \cdot \frac{\partial^{2}}{\partial t^{2}}\right) \psi+\frac{2\left(\alpha_{0}+\alpha_{1} \frac{\partial}{\partial t}\right)}{\left(\mu_{0}+\alpha_{0}\right)+\left(\mu_{1}+\alpha_{1}\right) \frac{\partial}{\partial t}} \chi+\frac{g}{\left(c_{2}^{2}+c_{2}^{\prime 2} \frac{\partial}{\partial t}\right)} \phi=0
$$

and

$$
\left(\nabla^{2}-\frac{4\left(\alpha_{0}+\alpha_{1} \frac{\partial}{\partial t}\right)}{\left(\nu_{0}+\epsilon_{0}\right)+\left(\nu_{1}+\epsilon_{1}\right) \frac{\partial}{\partial t}}-\frac{1}{c_{4}^{2}+c_{4}^{\prime 2} \frac{\partial}{\partial t}} \cdot \frac{\partial^{2}}{\partial t^{2}}\right) \chi-\frac{2\left(\alpha_{0}+\alpha_{1} \frac{\partial}{\partial t}\right)}{\left(\nu_{0}+\epsilon_{0}\right)+\left(\nu_{1}+\epsilon_{1}\right) \frac{\partial}{\partial t}} \cdot \nabla^{2} \psi=0
$$

where

$$
\begin{array}{lll}
c_{1}^{2}=\frac{2 \mu_{0}+\lambda_{0}}{\rho}, & c_{1}^{\prime 2}=\frac{2 \mu_{1}+\lambda_{1}}{\rho}, & c_{2}^{2}=\frac{\mu_{0}+\lambda_{0}}{\rho} \\
{c_{2}^{\prime}}^{2}=\frac{\mu_{1}+\lambda_{1}}{\rho}, & c_{4}^{2}=\frac{\nu_{0}+\epsilon_{0}}{\rho}, & c_{4}^{\prime 2}=\frac{\nu_{1}+\epsilon_{1}}{\rho} .
\end{array}
$$

From equations (2 8)-(2 10) we obtain

$$
\begin{gathered}
{\left[\left(\nabla^{2}-\frac{4\left(\alpha_{0}+\alpha_{1} \frac{\partial}{\partial t}\right)}{\left(\nu_{0}+\epsilon_{0}\right)+\left(\nu_{1}+\epsilon_{1}\right) \frac{\partial}{\partial t}}-\frac{1}{c_{4}^{2}+c_{4}^{\prime 2} \frac{\partial}{\partial t}} \cdot \frac{\partial^{2}}{\partial t^{2}}\right)\right.} \\
\quad\left\{\left(\nabla^{2}-\frac{1}{c_{1}^{2}+c_{1}^{\prime 2} \frac{\partial}{\partial t}} \cdot \frac{\partial^{2}}{\partial t^{2}}\right) \cdot\left(\nabla^{2}-\frac{1}{c_{2}^{2}+c_{2}^{\prime 2} \frac{\partial}{\partial t}} \cdot \frac{\partial^{2}}{\partial t^{2}}\right)\right. \\
\left.\quad+\frac{g^{2}}{\left(c_{1}^{2}+c_{1}^{\prime 2} \frac{\partial}{\partial t}\right)\left(c_{2}^{2}+c_{2}^{\prime 2} \frac{\partial}{\partial t}\right)} \cdot\left(\frac{\partial^{2}}{\partial r^{2}}+\frac{1}{r} \frac{\partial}{\partial r}\right)\right\} \\
\quad+\frac{2\left(\alpha_{0}+\alpha_{1} \frac{\partial}{\partial t}\right)}{\left(\mu_{0}+\alpha_{0}\right)+\left(\mu_{1}+\alpha_{1}\right) \frac{\partial}{\partial t}} \cdot \frac{2\left(\alpha_{0}+\alpha_{1} \frac{\partial}{\partial t}\right)}{\left(\nu_{0}+\epsilon_{0}\right)+\left(\nu_{1}+\epsilon_{1}\right) \frac{\partial}{\partial t}} \\
\left.\quad\left(\nabla^{2}-\frac{1}{c_{1}^{2}+c_{1}^{\prime 2} \frac{\partial}{\partial t}} \cdot \frac{\partial^{2}}{\partial t^{2}}\right) \nabla^{2}\right](\phi, \psi, \chi)=0 .
\end{gathered}
$$

\section{METHOD OF SOLUTION AND BOUNDARY CONDITIONS}

We apply the joint Fourier and Hankel transform (Debnath [5]) of zero order

$$
(\tilde{\bar{\phi}}, \tilde{\bar{\psi}}, \tilde{\chi})=\frac{1}{\sqrt{2 \pi}} \int_{-\infty}^{\infty} e^{\imath s t} d t \int_{0}^{\infty}(\phi, \psi, \chi) r J_{0}(k r) d r
$$

to $(211)$ and solve the transformed system subject to boundedness condition at infinity Thus it turns out that

$$
\tilde{\bar{\phi}}=\sum_{j=1}^{3} A_{\jmath} \exp \left(-\lambda_{\jmath} z\right), \quad \tilde{\bar{\psi}}=\sum_{\jmath=1}^{3} B j \exp \left(-\chi_{\jmath} z\right)
$$

and

$$
\tilde{\bar{\chi}}=\sum_{\jmath=1}^{3} C_{\jmath} \exp \left(-\lambda_{\jmath} z\right)
$$

where $\sum_{\jmath=1}^{3} \lambda_{\jmath}^{2}=\sum_{\jmath=1}^{3} k_{\jmath}^{2}-p q$

$$
\begin{aligned}
& \left(\lambda_{1} \lambda_{2}\right)^{2}+\left(\lambda_{2} \lambda_{3}\right)^{2}+\left(\lambda_{3} \lambda_{1}\right)^{2}=\left(k_{1} k_{2}\right)^{2}+\left(k_{2} k_{3}\right)^{2}+\left(k_{3} k_{1}\right)^{2}-k g^{3} k_{3}^{2}\left(c_{1}^{2}-i s c_{1}^{\prime 2}\right)^{-1}-p q\left(k_{1}^{2}+k^{2}\right) \\
& \left(\lambda_{1} \lambda_{2} \lambda_{3}\right)^{2}=\left(k_{1} k_{2} k_{3}\right)^{2}-k^{2} g^{2} k_{3}^{2}\left(c_{1}^{2}-i s c_{1}^{\prime 2}\right)^{-1}\left(c_{2}^{2}-i s c_{2}^{\prime 2}\right)^{-1}-p q k k_{1}^{2}
\end{aligned}
$$




$$
\begin{aligned}
& k_{1}^{2}=k^{2}-s^{2}\left(c_{1}^{2}-i s c_{1}^{\prime 2}\right)^{-1}, \quad k_{2}^{2}=k^{2}-s^{2}\left(c_{2}^{2}-i s c_{2}^{\prime 2}\right)^{1}, \\
& k_{3}^{2}=k^{2}+\nu_{0}^{2}-s^{2}\left(c_{3}^{2}-i s c_{3}^{\prime 2}\right)^{1}, \\
& p=\frac{2\left(\alpha_{0}-i s \alpha_{1}\right)}{\left(\mu_{0}+\alpha_{0}\right)-i s\left(\mu_{1}+\alpha_{1}\right)}, \quad q=\frac{2\left(\alpha_{0}-i s \alpha_{1}\right)}{\left(\nu_{0}+\epsilon_{0}\right)-i s\left(\nu_{1}+\epsilon_{1}\right)}, \quad \nu_{0}^{2}=2 q .
\end{aligned}
$$

The arbitrary constants $A_{\jmath}, B_{\jmath}$, and $C_{\jmath}$ are connected by the relations

$$
B_{\jmath}=p_{\jmath} A_{\jmath}, \quad C_{\jmath}=q_{\jmath} A_{\jmath}
$$

where

$$
\begin{aligned}
& p_{\jmath}=-\left(c_{1}^{2}-i s c_{1}^{2}\right)\left(\lambda_{\jmath}^{2}-k_{1}^{2}\right)\left(g k^{2}\right)^{-1} \\
& q_{\jmath}=-\frac{1}{p}\left[\left(\lambda_{\jmath}^{2}-k_{2}^{2}\right) p_{\jmath}+g\left(c_{2}^{2}-i s c_{2}^{\prime 2}\right)^{-1}\right] .
\end{aligned}
$$

The quantities $A_{j}$ involved in the solutions are determined from the boundary conditions

$$
\sigma_{z z}=-f_{1}(r, t), \quad \sigma_{z r}=-f_{2}(r, t), \quad \mu_{z \theta}=-f_{3}(r, t) \quad \text { on } \quad z=0
$$

where $f_{\imath}(r, t)>0$ for $i=1,2,3$, and

$$
\begin{aligned}
\sigma_{z z}=2\left(\mu_{0}+\mu_{1} \frac{\partial}{\partial t}\right) \frac{\partial^{2} \phi}{\partial z^{2}}+ & \left(\lambda_{0}+\lambda_{1} \frac{\partial}{\partial t}\right)\left(\frac{\partial^{2}}{\partial r^{2}}+\frac{1}{r} \frac{\partial}{\partial r}+\frac{\partial^{2}}{\partial z^{2}}\right) \phi \\
& -2\left(\mu_{0}+\mu_{1} \frac{\partial}{\partial t}\right) \frac{\partial}{\partial z}\left(\frac{\partial^{2}}{\partial r^{2}}+\frac{1}{r} \frac{\partial}{\partial r}\right) \psi \\
\sigma_{z r}=2\left(\mu_{0}+\mu_{1} \frac{\partial}{\partial t}\right) \frac{\partial^{2} \phi}{\partial z \partial r}+\left\{\left(\mu_{0}+\alpha_{0}\right)+\left(\mu_{1}+\alpha_{1}\right) \frac{\partial}{\partial t}\right\} \frac{\partial^{3} \psi}{\partial r \partial z^{2}} & -2\left\{\left(\mu_{0}-\alpha\right)+\left(\mu_{1}-\alpha_{1}\right) \frac{\partial}{\partial t}\right\} \frac{\partial}{\partial r}\left(\frac{\partial^{2}}{\partial r^{2}}+\frac{1}{r} \frac{\partial}{\partial r}\right) \psi+2\left(\alpha_{0}+\alpha_{1} \frac{\partial}{\partial t}\right) \frac{\partial \chi}{\partial r}
\end{aligned}
$$

and

$$
\mu_{z \phi}=-\left\{\left(\nu_{0}+\epsilon_{0}\right)+\left(\nu_{1}+\epsilon_{1}\right) \frac{\partial}{\partial t}\right\} \frac{\partial^{2} \chi}{\partial r \partial z} .
$$

The quantities $A$, found from the boundary conditions ( 33 ) are as follows

$$
A_{\jmath}=(-1)^{\jmath} \frac{\triangle_{\jmath}}{\triangle}
$$

where

$$
\begin{aligned}
\triangle_{1} & =f_{1}\left(b_{2} c_{3}-b_{3} c_{2}\right)+f_{2}\left(c_{2} a_{3}-c_{3} a_{2}\right)+f_{3}\left(a_{2} b_{3}-a_{3} b_{2}\right) \\
\triangle_{2} & =f_{1}\left(b_{1} c_{3}-b_{3} c_{1}\right)+f_{2}\left(c_{1} a_{3}-c_{3} a_{1}\right)+f_{3}\left(a_{1} b_{3}-a_{3} b_{1}\right) \\
\triangle_{3} & =f_{1}\left(b_{1} c_{2}-b_{2} c_{1}\right)+f_{2}\left(c_{1} a_{2}-c_{2} a_{1}\right)+f_{3}\left(a_{1} b_{2}-a_{2} b_{1}\right) \\
\triangle & =a_{1}\left(b_{2} c_{3}-b_{3} c_{2}\right)+a_{2}\left(b_{3} c_{1}-b_{1} c_{3}\right)+a_{3}\left(b_{1} c_{2}-b_{2} c_{1}\right) \\
a_{\jmath}= & 2\left(\mu_{0}-i s \mu_{1}\right) \lambda_{\jmath}^{2}+\left(\lambda_{0}-i s \mu_{1}\right)\left(\lambda_{\jmath}^{2}-k^{2}\right)-2\left(\mu_{0}-i s \mu_{1}\right) p_{\jmath} \lambda_{\jmath} k^{2} \\
b_{\jmath}= & 2\left(\mu_{0}-i s \mu_{1}\right) \lambda_{\jmath} k-k\left[\left(\mu_{0}+\alpha_{0}\right)-i s\left(\mu_{1}+\alpha_{1}\right)\right\} \lambda_{\jmath}^{2}+\left\{\left(\mu_{0}-\alpha_{0}\right)\right. \\
& \left.\left.\quad-i s\left(\mu_{1}-\alpha_{1}\right) k^{2}\right\}\right] p_{\jmath}+2\left(\alpha_{0}-i s \alpha_{1}\right) k q_{\jmath}
\end{aligned}
$$




$$
c_{J}=\left[\left(\nu_{0}+\epsilon_{0}\right)-\imath s\left(\nu_{1}+\epsilon_{1}\right)\right] \lambda_{\jmath} q_{J} k .
$$

In view of the inverse Fourier and Hankel transformations combined with relations (24)-(2 6) and (2 7ab) we get

$$
\begin{aligned}
& u_{r}=-\frac{1}{\sqrt{2 \pi}} \int_{\infty}^{x} e^{\imath s t} d s \int_{0}^{\infty} \sum_{j}^{3}\left(1-\lambda_{\jmath} p_{\jmath}\right) a_{\jmath} \exp \left(-\lambda_{\jmath} z\right) k^{2} J_{1}(k r) d k \\
& \omega_{\theta}=\frac{1}{\sqrt{2 \pi}} \int_{x_{i}}^{\infty} e^{\imath s t} d s \int_{0}^{\infty} \sum_{j=1}^{3}\left\{q_{\jmath} A_{\jmath} \exp \left(-\lambda_{\jmath} z\right)\right\} k^{2} J_{1}(k r) d k
\end{aligned}
$$

and

$$
u_{z}=-\frac{1}{\sqrt{2 \pi}} \int_{-\infty}^{\infty} e^{-\imath s t} d s \int_{0}^{\infty} \sum_{\jmath=1}^{3}\left(\lambda_{\jmath}-k^{2} p_{\jmath}\right) A_{\jmath} \exp \left(-\lambda_{\jmath} z\right) k J_{0}(k r) d k,
$$

where $A_{\jmath}$ are given by (3 4) Hence, utilizing results $\left(\begin{array}{ll}2 & 1\end{array}\right)-(22)$ we can find the state of strain and the state of stress in the semi infinite space

When the viscosity and gravity are not taken into account, that is, when $\lambda_{1}, \mu_{1}, \alpha_{1}, \beta_{1}, \gamma_{1}, \epsilon_{1}$ are .equal to zero and $g=0$, relations $(35)-(37)$ for displacement components and rotation component reduce to

$$
\begin{aligned}
& u_{r}=-\frac{1}{\sqrt{2 \pi}} \int_{-\infty}^{\infty} e^{-\imath s t} d s \int_{0}^{\infty} k^{2}\left\{A_{1} \exp \left(-\lambda_{1} z\right)-\sum_{\jmath=2}^{3} A_{\jmath} \exp \left(-\lambda_{\jmath} z\right)\right\} J_{1}(k r) d k \\
& \omega_{\theta}=\frac{1}{\sqrt{2 \pi}} \int_{-\infty}^{\infty} e^{-\imath s t} d s \int_{0}^{\infty} \sum_{\jmath=2}^{3} x \lambda_{\jmath} A_{\jmath} \exp \left(-\lambda_{\jmath} z\right) k^{2} J_{1}(k r) d k
\end{aligned}
$$

and

$$
u_{z}=-\frac{1}{\sqrt{2 \pi}} \int_{-\infty}^{\infty} e^{-\imath s t} d s \int_{0}^{\infty} k\left\{\lambda_{1} A_{1} \exp \left(-\lambda_{1} z\right)-k^{2} \sum_{\jmath=2}^{3} A_{\jmath} \exp \left(-\lambda_{\jmath} z\right)\right\} J_{0}(k r) d k
$$

where

$$
\begin{array}{rlrl}
a_{1} & =2 \mu_{0} \lambda_{1}^{2}+\lambda_{0}\left(\lambda_{1}^{2}-k^{2}\right), \quad a_{\jmath}=-2 \mu_{0} k^{2} \lambda_{\jmath}, \quad(j=2,3) \\
c_{\jmath} & =\left(\nu_{0}+\epsilon_{0}\right) k \lambda_{\jmath} q_{\jmath}, & q_{\jmath}=x \lambda_{\jmath}=-\frac{1}{p}\left(\lambda_{\jmath}^{2}-k_{2}^{2}\right) \\
\lambda_{1}^{2} & =k^{2}-\frac{s^{2}}{c_{1}^{2}}, & \lambda_{2}^{2}+\lambda_{3}^{2}=k_{2}^{2}+k_{3}^{2}-p_{0} s_{0} \\
\lambda_{2}^{2} \lambda_{3}^{2} & =k_{2}^{2} k_{3}^{2}-p_{0} s_{0} k^{2}, \quad p_{0}=\frac{2 \alpha_{0}}{\mu_{0}+\alpha_{0}}, \quad s_{0}=\frac{2 \alpha_{0}}{\gamma_{0}+\epsilon_{0}} .
\end{array}
$$

Relations (3 8)-(3 10) are in agreement with those obtained by Nowacki and Nowacki [4]

\section{PARTICULAR CASE}

We now consider a particular case of loading on the semi-infinite space boundary, that is, the loading oscillating harmonically in time, the medium being stationary for $t<0$

The boundary conditions on the surface $z=0$ are

$$
\sigma_{z z}=Q e^{-\imath \omega t} f(r), \quad \sigma_{z r}=0, \quad \mu_{z \theta}=0 .
$$

Now the constants $A_{\jmath}$ in the equations (3 4) reduce to 


$$
A_{J}=(-1)^{\jmath} \frac{\triangle_{j}^{0}}{\triangle}
$$

where

$$
\triangle_{1}^{0}=\tilde{\tilde{f}}\left(b_{2} c_{3}-b_{3} c_{2}\right), \quad \triangle_{2}^{0}=\tilde{\tilde{f}}\left(b_{1} c_{3}-b_{33} c_{1}\right), \quad \triangle_{3}^{0}=\tilde{\tilde{f}}\left(b_{1} c_{2}-b_{2} c_{1}\right)
$$

and

$$
\tilde{\bar{f}}(s, k)=\frac{1}{\sqrt{2 \pi}} \int_{\infty}^{x^{\prime}} e^{\iota \iota t} d t \int_{0}^{x} f(r) e^{-\imath \omega t} r J_{0}(k r) d r=\sqrt{2 \pi} \delta(s-\omega) \tilde{\bar{f}}(k)
$$

Putting $f(r)=F_{0} \sin \xi r$, we have

$$
\bar{f}(k)=-\frac{d}{d \xi}\left\{\frac{H(k-\xi)}{\sqrt{k^{2}-\xi^{2}}}\right\} .
$$

Thus it turns out that

$$
\begin{aligned}
& u_{r}=e^{-\imath \omega t} \frac{d}{d \xi} \int_{\xi}^{\infty} k^{2} \sum_{\jmath=1}^{3}\left(1-\lambda_{\jmath}^{\prime} p_{\jmath}^{\prime}\right) A_{\jmath}^{\prime} \cdot\left(k^{2}-\xi^{2}\right)^{-\frac{1}{2}} \exp \left(-\lambda_{\jmath}^{\prime} z\right) J_{1}(k r) d k \\
& u_{z}=e^{-\imath \omega t} \frac{d}{d \xi} \int_{a}^{\infty} k \sum_{\jmath=1}^{3}\left(\lambda_{\jmath}^{\prime 2}-k^{2} p_{\jmath}^{\prime}\right) A_{\jmath}^{\prime} \cdot\left(k^{2}-\xi^{2}\right)^{-\frac{1}{2}} \exp \left(-\lambda_{\jmath}^{\prime} z\right) J_{0}(k r) d k \\
& \omega_{\theta}=-e^{-\imath \omega t} \int_{a}^{\infty} k^{2}\left(\sum_{\jmath=1}^{3} q_{\jmath}^{\prime} A_{\jmath}^{\prime}\right)\left(k^{2}-\xi^{2}\right)^{-\frac{1}{2}} \exp \left(-\lambda_{\jmath}^{\prime} z\right) J_{1}(k r) d k
\end{aligned}
$$

where dashed quantities represent the value of the function at $s=\omega$

Results (3 8)-(3 10) show the striking difference between the displacement and rotation due to the influences of gravity when the effects of viscosity and gravity are neglected In the absence of gravity, $\lambda_{1}^{2}, \lambda_{2}^{2}, \lambda_{3}^{2}$ are given in (311), and these quantities are given in Section 3 where the effects of gravity and viscosity are included

In conclusion, we state that $\lambda_{\jmath}$ for the present case also depend on gravity and the corresponding results are changed from those where the effects of gravity and viscosity are neglected Further, the displacement field and rotation are correspondingly modified with increasing depth The modification is due to the pressure of viscosity and gravity Finally, in the absence of gravity with very small viscosity, the results reduce to those of the classical theory of elasticity due to Ghosh $[6,7]$

\section{REFERENCES}

[1] LOVE, A E H., Some Problems of Geodynamics, Dover Publications, New York

[2] DE, S N. and SENGUPTA, P R , Jour. Acous. Soc. Amer. 55 (1974) 5-10

[3] BIOT, M A , Mechanics of Incremental Deformatıons, John Wiley (1965), 273-281

[4] NOWACKI, W and NOWACKI, W K, Proc. Vib. Prob. 2 (1969), 10-17

[5] DEBNATH, L, Integral Transforms and Their Applicatıons, CRC Press, Boca Raton (1995)

[6] GHOSH, B C, Bull. Polon. Acad. Scl. Tech. 32 (1984), 7-8

[7] SENGUPTA, P R and B C GHOSH, Acad. Polon. Scl. Tech. 24 (1978), 251-262 


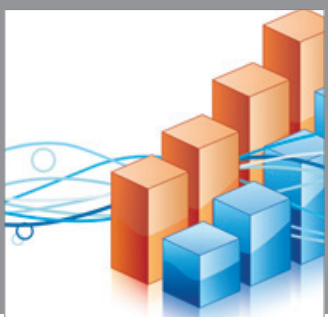

Advances in

Operations Research

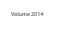

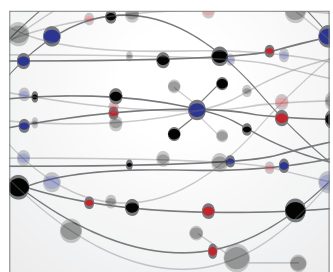

\section{The Scientific} World Journal
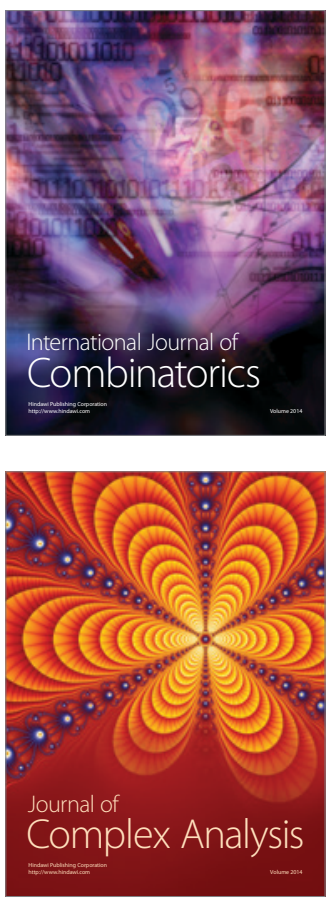

International Journal of

Mathematics and

Mathematical

Sciences
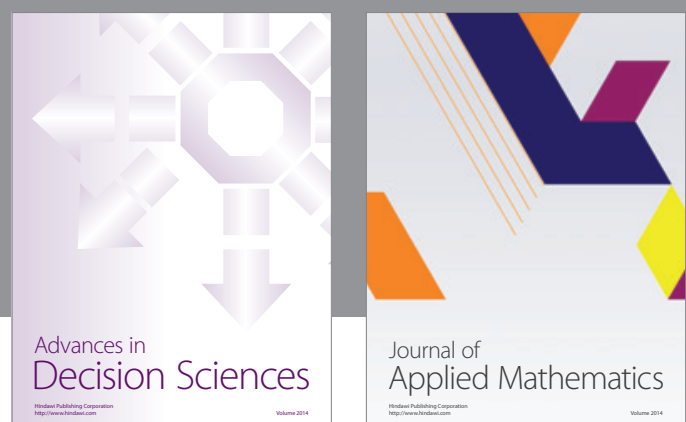

Journal of

Applied Mathematics
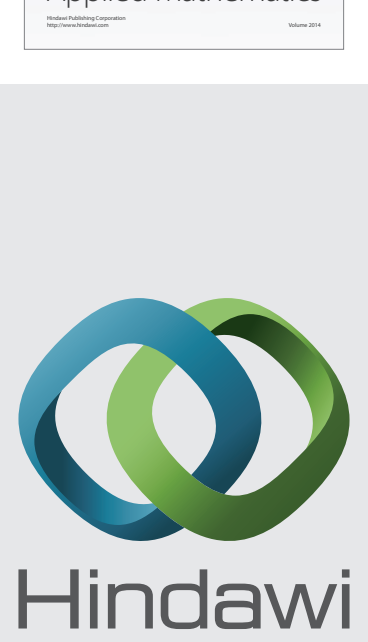

Submit your manuscripts at http://www.hindawi.com
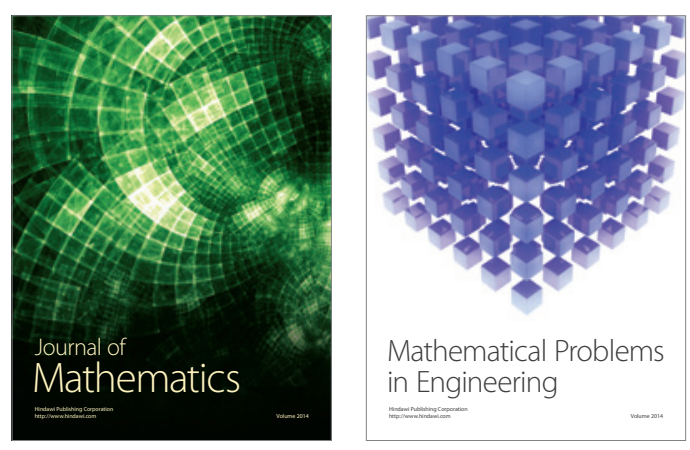

Mathematical Problems in Engineering
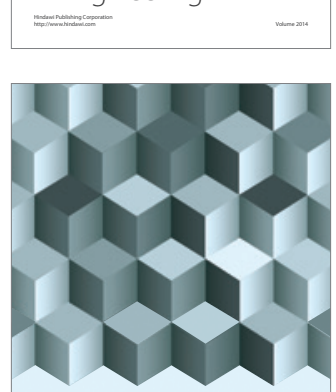

Journal of

Function Spaces
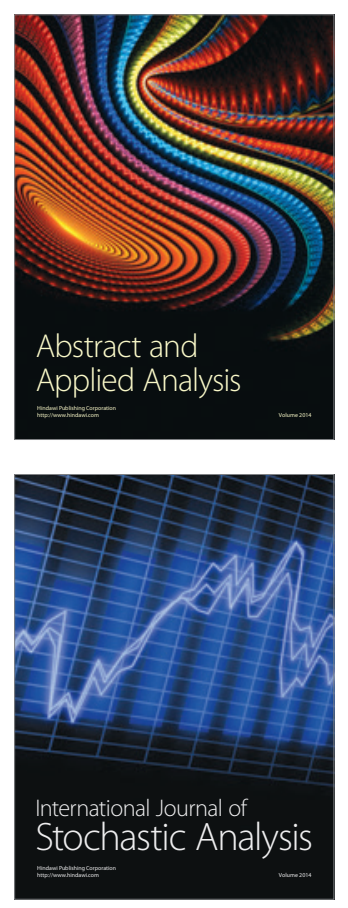

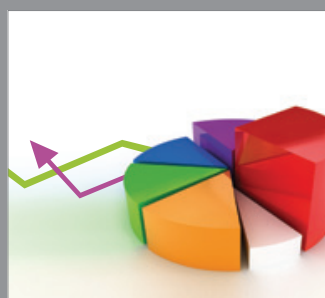

ournal of

Probability and Statistics

Promensencen
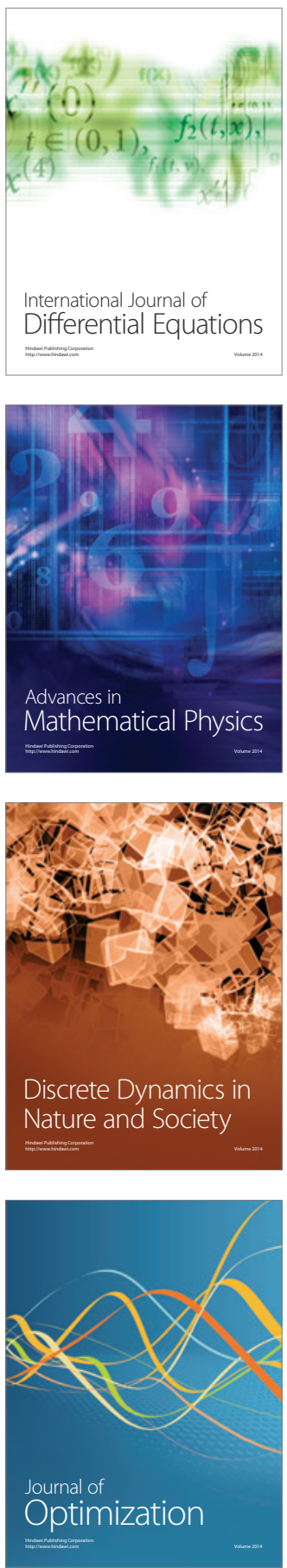